\title{
IMRT Commissioning and Verification Measurements on Siemens (ARTISTE) Linear Accelerator
}

\author{
Tamer Dawod ${ }^{1}$, Yousri Rostom², Mahmoud Abouzeid ${ }^{3}$ and Mostafa Mosad ${ }^{1}$ \\ ${ }^{1}$ Clinical Oncology and Nuclear Med. Department, Fac. Of Medicine, Mansoura University, ${ }^{2}$ Clinical \\ Oncology and Nuclear Med. Department, Fac. Of Medicine, Alexandria University and ${ }^{3}$ Physics \\ Department, Fac. Of Science, Mansoura University.
}

\begin{abstract}
This study carried out in Ayadi Almostkbal Oncology Center - Alexandria, measurements carried on siemens linear accelerator (ARTISTE) which produces photons with energy 6 and $10 \mathrm{MV}$.

This study aimed to dosimetric and verification measurements to apply step-and-shoot intensity modulated radiation therapy (IMRT) technique on patients. IMRT is a technology in radiation oncology that delivers radiation more precisely to the tumor while relatively sparing the surrounding normal tissues.

Dosimetric measurements were carried out by using linear accelerator siemens (ARTISTE), PTW dosimetry system (relative and absolute) and slab solid phantom, using this tools we measure percentage depth dose, beam profile, flatness and symmetry. The measured data was collected and entered (transferred) to the treatment planning system CMS (XIO) for calculating dose distribution.

These measurements were verified by comparing dose distribution came out planning system with dose distribution measured by PTW 2D-Array on linear accelerator.

Dosimetry involves more than accounting for delivered dose. It involves ensuring that the MLC shapes are accurate and are correctly assigned to the patient. Any changes in shaping must be correctly and accurately incorporated into the field sequence. The information system should be implemented in a way that ensures good quality assurance practices.
\end{abstract}

Key words: IMRT, MLC, 2D-Array, dosimetry, radiation oncology.

Corresponding Author: Tamer Dawod

E-mail: tamerdawod@gmail.com

\section{INTRODUCTION}

Intensity modulated radiation therapy (IMRT) is a technology in radiation oncology that delivers radiation more precisely to the tumor while relatively sparing the surrounding normal tissues ${ }^{1-3}$. It also introduces concepts of inverse planning and computer-controlled radiation deposition and normal tissue avoidance in contrast to the conventional trial-and-error approach ${ }^{4}$. IMRT became one of the most important treatment techniques in radiation oncology because of its ability to create multiple targets and multiple avoidance structures, to treat different targets simultaneously to different doses, as well as to weight targets and avoidance structures according to their importance ${ }^{5}$. By delivering radiation with greater precision, IMRT has been shown to minimize acute treatment-related morbidity, making dose escalation feasible which may ultimately improve local tumor control ${ }^{6}$.

Intensity modulated radiation therapy (IMRT) is an extension of 3D conformal radiation therapy (3DCRT). Thus, there are new capabilities of linear accelerators and collimators that need to be installed, commissioned, and maintained. Also, computing the needed intensity patterns and machine instructions to create them into the treatment planning system. The computer algorithms associated with the planning system has to be commissioned for dose calculation accuracy ${ }^{7}$.

In general IMRT requires the use of the socalled "inverse" treatment planning system that work backwards from a desired dose distribution to the fluence distribution needed to approximate those dose distributions. Inverse planning system is not truly inverse, because this is mathematically impossible, inverse planning programs are only able to approximate the desired dose distributions with physical fluence patterns ${ }^{8}$.

The Multileaf position calibration is the single most important mechanical QA item for MLC-based IMRT delivery. One of the few calibration tests that should be measured is the gap between leaves when the leaves were supposed to be closed or nearly closed. One important consideration for MLCs with rounded leaf ends is the 


Vol. 8 | No. 1-2 $2012 \quad$ Tamer Dawod et al.

position difference between the radiation field edge and the light field edge (acting as surrogate for line between the radiation source and the tangent to the rounded leaf end). Because of the leaf geometry with rounded leaf ends, this position difference can be significant. For IMRT, this difference leads to significant dose delivery errors if not properly modeled by the treatment planning system. The tests of the leaf calibration accuracy have typically relied on the irradiation of radiographic film using a specific radiation pattern that relies on the interaction of the steep penumbra and the sensitivity of the abutment dose on calibration errors [9].

\section{MATERIALS AND METHODS}

This study was carried out using the measuring equipment's and irradiation facilities, namely: Siemens Linac (ARTISTE), CMS (Xio 4.5) 3D planning system, PTW dosimetry system, 2D-array dosimeter and Slab solid phantom, used for all measurements required for verifications, QA, and analysis.

The central axis depth doses were measured using a commercially available PTW water scanning system. The radiation of photon beam was detected via two Semiflex ionization chambers $\left(0.125 \mathrm{~cm}^{3}\right)$. For $6 \mathrm{MV}$ energy, the measurements were made at $100 \mathrm{~cm}$ FSD for different open square field sizes in the range from $2 \times 2$ to $40 \times 40 \mathrm{~cm}^{2}$ and depths from 0.0 to $35.0 \mathrm{~cm}$ with increment $1.0 \mathrm{~mm}$ and $2.0 \mathrm{~mm}$ along the central axis. The data for every field size were normalized to $100 \%$ at the depth of maximum dose dmax (reference depth at $10 \mathrm{~cm} \times 10 \mathrm{~cm}$ field size), which corresponds to $1.5 \mathrm{~cm}^{10}$.

Beam profiles measurements were also used in conjunction with percentage depth doses in the calculation of isodose distributions. The measurements were experimentally determined using $0.125 \mathrm{~cm}^{3}$ ionization chamber as in the case of depth dose measurements except in which the scanning ionization chamber moves transversely along $\mathrm{x}$ and $\mathrm{y}$ directions of the water phantom. The ionization chamber was scanned across the radiation field of square sides $5 \times 5 \mathrm{~cm}$ and $30 \times 30 \mathrm{~cm}^{2}$ with increment $2 \mathrm{~mm}$. The beam profiles were measured at depths in the range from dmax to $30 \mathrm{~cm}$ in a water phantom for each field size with a constant FSD of $100 \mathrm{~cm}$.

Beam profile parameters were obtained by MEPHYSTO software that is used with automatic scanning system. The beam parameters, homogeneity (flatness), symmetry and penumbra were measured for both longitudinal and transverse axis of each field size at different depths. They were referring to the flattened region of the beam profile scan.
The flatness was calculated at field size measured with the $50 \%$ of the dose along the central axis, which is known as radiological field width ${ }^{11}$. Dmax and Dmin are the maximum and minimum dose within the flattened region referred to the dose value at the central axis respectively.

Symmetry was a measure of whether the beam's dose consistent on each side of the beam profile and is defined as the being maximum ratio within the flattened region, multiplied with $100^{12,13}$.

Total Scatter Factors (TSCFs) [14] was Measured in water at $\mathrm{SSD}=100 \mathrm{~cm}$ with pinpoint ionization chamber at reference depth $(10 \mathrm{~cm})$ for collimator setting of : $1 \times 1,2 \times 2,3 \times 3,4 \times 4,5 \times 5,6 \times 6,8 \times 8,10 \times 10$, $12 \times 12,15 \times 15,18 \times 18,20 \times 20,25 \times 25,30 \times 30,35 \times 35$, $40 \times 40 \mathrm{~cm}^{2}$. The measurements for field sizes from $5 \times 5 \mathrm{~cm}^{2}$ to $40 \times 40 \mathrm{~cm}^{2}$ were repeated and verified by $0.6 \mathrm{~cm}^{3}$ ionization chamber. Absolute output for reference field size $\left(10 \times 10 \mathrm{~cm}^{2}\right)$, was measured at reference depth $(10 \mathrm{~cm})$ employing the same setup used to collect TSCF.

Collimator Factor $(\mathrm{Sc})^{15}$ measurement was carried out in air (using mini-phantom) at $\mathrm{SSD}=100 \mathrm{~cm}$ with (Pinpoint) ionization chamber at reference depth $(10 \mathrm{~cm})$ at isocenter for the collimator settings of: $1 \times 1$, $2 \times 2,3 \times 3,4 \times 4,5 \times 5,6 \times 6,8 \times 8,10 \times 10,12 \times 12,15 \times 15$, $18 \times 18,20 \times 20,25 \times 25,30 \times 30,35 \times 35$, and $40 \times 40 \mathrm{~cm}^{2}$.

Phantom Scatter Correction Factor (PSCFs) was computed from (Total Scatter Factor / Collimator factor) above.

Physical Wedge Transmission Factor was measured in water phantom at $\mathrm{SSD}=100 \mathrm{~cm}$ using $(0.6 \mathrm{cc})$ chamber at reference depth $(10 \mathrm{~cm})$ for collimator setting of: $5 \times 5,10 \times 10,15 \times 15,20 \times 20,25 \times 25 \mathrm{~cm}^{2}$ and maximum wedged square field size. Physical Wedge transmission factors were also calculated as a ratio against standard field size $10 \times 10 \mathrm{~cm}^{2}$.

Collimator Transmission was measured by using a mini-phantom ${ }^{16}$ :

Measuring the dose rate on the CAX of the beam for the smallest collimator setting at isocenter that covers the entire mini-phantom.

Close the width jaws down to as close to zero as obtainable and move the mini-phantom as needed to make sure no beam is intercepting the phantom and measure the dose rate.

The PTW 2D-Array (seven 29) in combination with the VeriSoft analysis software were used as a dosimetric verification tool for IMRT fields as the following ${ }^{17}$ : 
- Determining the MUs for 1 Gy with $6 \mathrm{MV}$ photons by $10 \times 10 \mathrm{~cm}$ field size. Absolute dose was measured at $10 \mathrm{~cm}$ depth in RW3 slab phantom at $\mathrm{FSD}=90.8 \mathrm{~cm}(5 \mathrm{~cm}$ was used as backscatter material).

- The 2D-array was put into RW3 phantom and adjusted to have an effective measuring depth of $10 \mathrm{~cm}$.

- Calibration factor was determined by dose coming from the reading of the array chambers on the central beam axis for $1 \mathrm{~Gy}$ irradiation.

- A plan of 10x10 field size at $10 \mathrm{~cm}$ depth by the same setup conditions was carried out by using the treatment planning system.

- The planning dose cube IMRT plan was exported via dicom format to PTW Verisoft on 2D-Array and compared with the corresponding measured intensity map (gamma test) and applying the correction factors between planning system and absolute dosimetry and between the 2D-Array and absolute dosimetry.

- The Gamma Index Analysis is the most common method to compare two IMRT matrices with respect to dose difference and a physical distance between the comparison point $r^{18}$.

$$
\gamma\left(\mathbf{r}_{m}\right)=\min \left\{\Gamma\left(\mathbf{r}_{m}, \mathbf{r}_{c}\right)\right\} \forall\left\{\mathbf{r}_{c}\right\}
$$

Where

$$
\begin{aligned}
& \Gamma\left(\mathbf{r}_{m}, \mathbf{r}_{c}\right)=\sqrt{\frac{r^{2}\left(\mathbf{r}_{m}, \mathbf{r}_{c}\right)}{\Delta d_{M}^{2}}+\frac{\delta^{2}\left(\mathbf{r}_{m}, \mathbf{r}_{c}\right)}{\Delta D_{M}^{2}}} \\
& r\left(\mathbf{r}_{m}, \mathbf{r}_{c}\right)=\left|\mathbf{r}_{c}-\mathbf{r}_{m}\right|
\end{aligned}
$$

And

$$
\delta\left(\mathbf{r}_{m}, \mathbf{r}_{c}\right)=D_{c}\left(\mathbf{r}_{c}\right)-D_{m}\left(\mathbf{r}_{m}\right)
$$

Is the difference between dose values on the calculated and measured distributions, respectively the pass-fail criteria therefore become fails.

$\mathrm{V}(\mathrm{rm}) \leq 1$, calculation passes, $\mathrm{V}(\mathrm{rm})>1$, calculation

- After that the profile should be alright, if this does not work, then there is a problem somewhere.

\section{RESULTS}

\section{Commissioning data:}

Figure (1) illustrates the influence of the open field sizes on the percent depth dose curves. They are characterized by the buildup of dose at the surface reaching a maximum dose, then the dose decreases as the photon beam traverse through the phantom ${ }^{19,20}$. The percent depth dose increases with the field size due to the increase in scattered radiation at larger field sizes. In addition, it is obvious from the Figure (1) that the surface dose increases with the field size because of the increase in scattered radiation. The depth of maximum dose was found to be at $1.5 \pm 0.1 \mathrm{~cm}$ for $6 \mathrm{MV}^{21}$ photon beam over the range of field sizes from $2 \times 2$ to $40 \times 40 \mathrm{~cm}^{2}$. After the radiation beam has penetrated to the dmax, there is a gradual decrease in the dose $\mathrm{e}^{22}$.

Figures (2) and (3) show an open beam profile measurements of $6 \mathrm{MV}$ Siemens (ARTISTE) Linac. Across the radiation field $2 \times 2 \mathrm{~cm}^{2}$ and $10 \times 10 \mathrm{~cm}^{2}$ at depths ranged from dmax to $30 \mathrm{~cm}$.

The resulting beam profile scans demonstrate the variation in flatness and symmetry of the field size as a function of the specified depths $(1.5,5,10,15,20,25$ and $30 \mathrm{~cm})^{23}$. The beam homogeneity increases with the field size and depth in which affecting in the beam uniformity ${ }^{24}$. This increase in the homogeneity value is illustrated in more detail in Figure (4), in this figure the beam profiles for field size $10 \times 10 \mathrm{~cm}^{2}$ at depths $1.5,10$, and $20 \mathrm{~cm}$ are normalized to the dose at the center of the field, thus obtaining an off-axis ratio (OAR) for each off axis distance.

Additional measured parameters including Total Scatter Factors (TSCFs), Collimator Factors (Sc) ${ }^{25}$ and Phantom Scatter Correction Factors ${ }^{26}$ (PSCFs) are shown in Table (1).

\section{Verification measurements:}

The verification method adopted here is to transfer the CT based treatment plan to a homogenous phantom. The plan is then recalculated in the TPS for this phantom. Measurements were performed in the phantom by 2D-Array. The calculated dose distribution in the phantom is compared with measurements in the phantom. The dose distributions calculation in the phantom must be exactly the same beam and MLC settings were used as in the patient case (all angles for the plan were reset to zero degree to be measured using 2D- array). If the measurements and the calculations in the phantom are within acceptable tolerances, the IMRT plan is considered to be accurate to deliver to the patient ${ }^{17,27-30}$. When an IMRT treatment plan has been accepted for patient treatment, it has to be verified according to the description above.

IMRT mainly used for treatments of the prostate, head and neck tumors and for irradiations of the breast if the internal mammary lymph node chain has to be included into the target volume. Figure (5) shows a comparison between measured and calculated plan for verification of real patient IMRT plan (prostate). 
According to the tolerance values for homogeneous simple fields, the penumbra region should be within $2 \mathrm{~mm}$ or $10 \%$. By just studying the profiles by eye it is hard to say, especially in the z-direction in the penumbra region, if the result is within the tolerance. A gamma evaluation with $3 \%$ and $3 \mathrm{~mm}$ criteria is shown in Figure (5), revealing that it is only in the penumbra region that acceptance fails. The colours of the palette range are set to be green for $100 \%(\gamma=1)$, and accepted regions are green and most yellow. Regions that fail are shown in red. The gamma test evaluation method is not a good tool for evaluation of low dose regions, where the

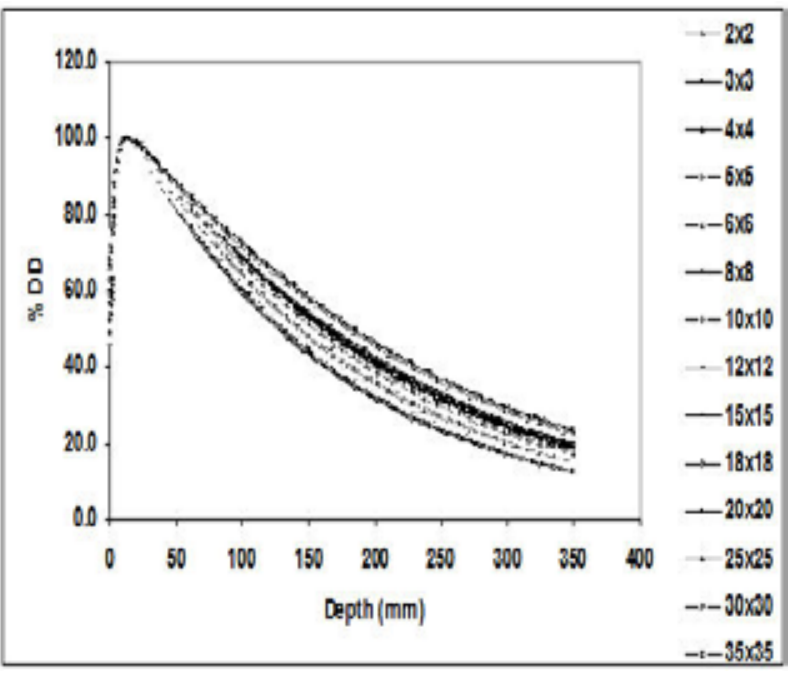

Figure 1: PDDs of $6 \mathrm{MV}$ photon beam for open square fields sizes at $\mathrm{FSD}=100 \mathrm{~cm}$ in water phantom.

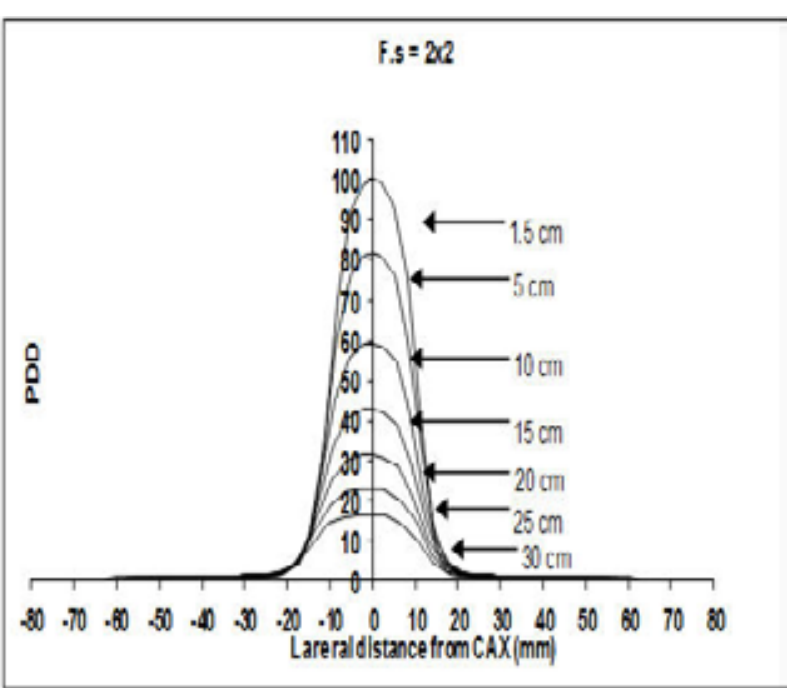

Figure 2: Beam profiles of $6 \mathrm{MV}$ photon beam for $\left(2 \times 2 \mathrm{~cm}^{2}\right)$ field size at depths of $(1.5,5,10,15,20,25$ and $30 \mathrm{~cm})$. calculation can fail though it is within the set criteria. For example if we are comparing two dose points of $4 \%$ and $1 \%$ dose, and the dose criteria is set to be $2 \%$, this will lead to a gamma value larger than $1(4 \%-1 \%) / 2 \%)$. The $3 \%$ dose difference can still be within acceptable tolerances but the gamma calculation fails.

Five IMRT fields with a total of 72 segments for a real prostate patient were used for comparison and verification. The results presented in Figure (5) indicate quite good agreement between measured and TPS calculated doses.

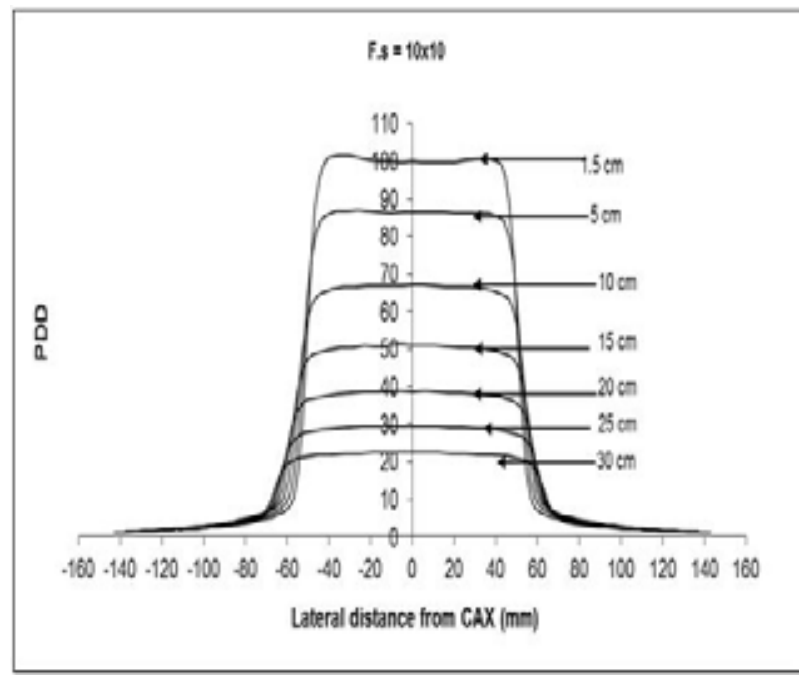

Figure 3: Beam profiles of $6 \mathrm{MV}$ photon beam for $\left(10 \times 10 \mathrm{~cm}^{2}\right)$ field size at depths of $(1.5,5,10,15,20,25$ and $30 \mathrm{~cm})$.

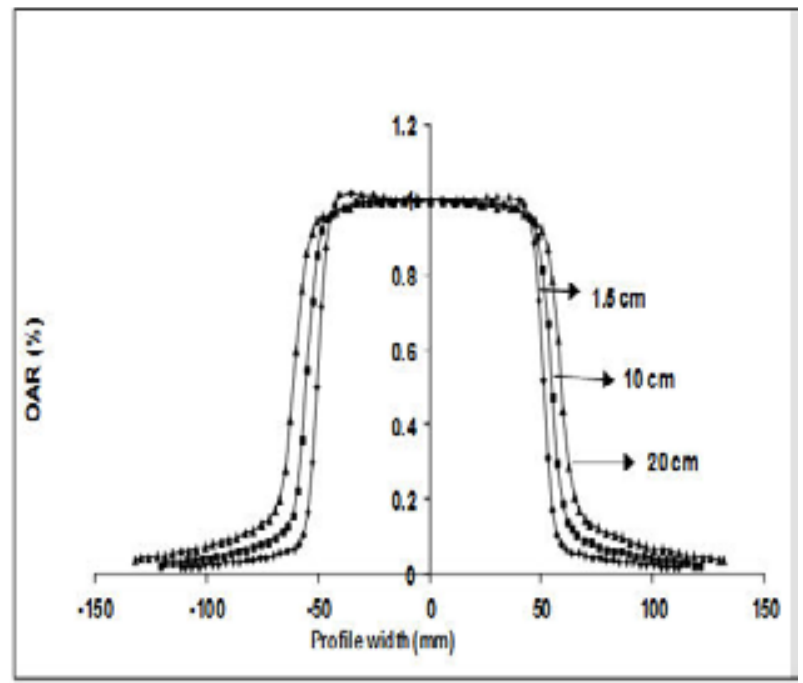

Figure 4: Dose profiles for $\left(10 \times 10 \mathrm{~cm}^{2}\right)$ at $1.5,10$, and $20 \mathrm{~cm}$ depth normalized to unity at the center of the field for $6 \mathrm{MV}$ photon beam. 
Kasr-El-Aini Journal Of Clinical Oncology And Nuclear Medicine

Vol. 8 | No. 1-2 $2012 \quad$ IMRT Commissioning and Verification Measurements on Siemens (ARTISTE)

\begin{tabular}{|c|c|c|}
\hline Gamma 2D-Parameters & Setings & \\
\hline $30 \mathrm{~min}$ Distance- To-Agreentient & Pariegctede & Grinas 51 \\
\hline 30\% Dose biterence with ref. to local dose & Geth & $9203>10003$ \\
\hline Suppress doses below $30 \%$ of max, Goee of mesured daba set & Ielew & 738564003 \\
\hline
\end{tabular}
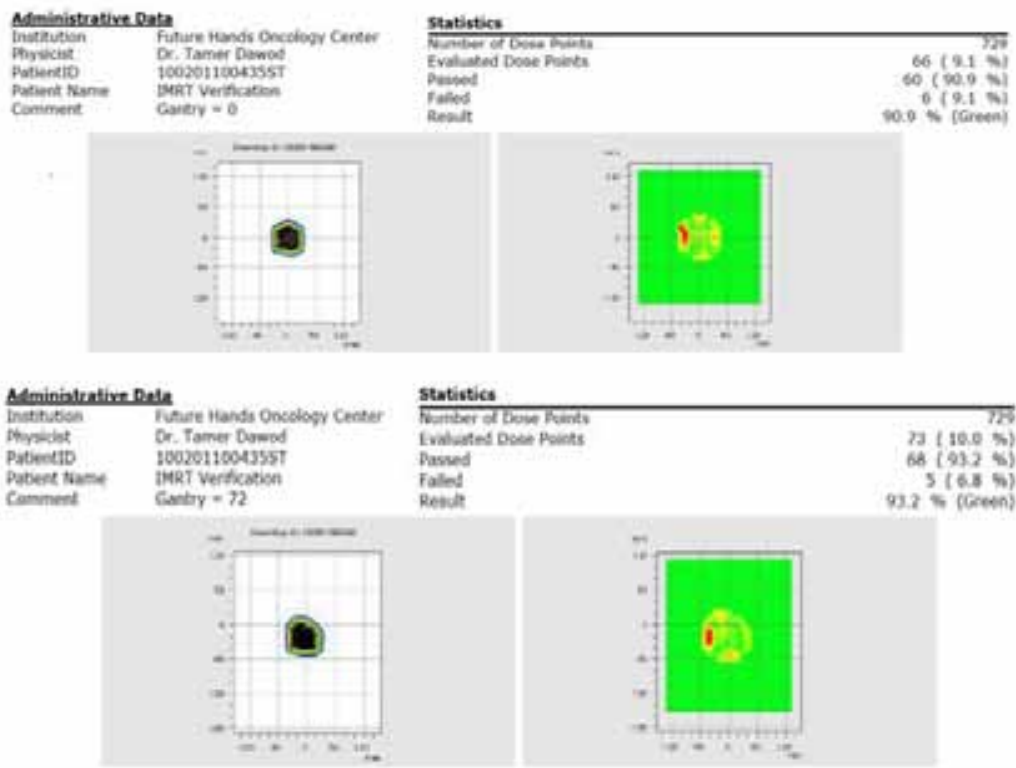

Faled

$5(6,5)$
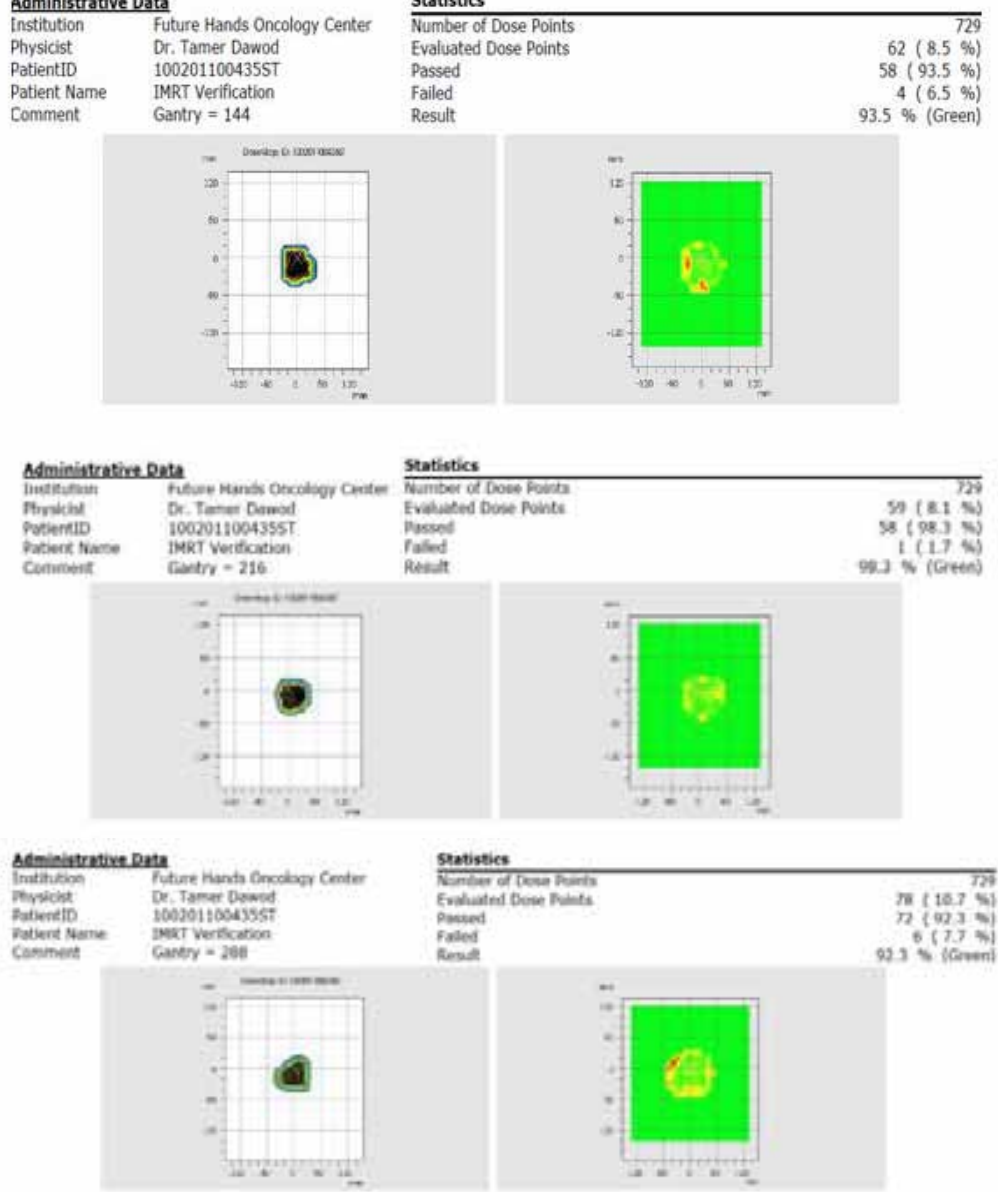

Figure 5: All fields of IMRT real prostate patient isodose verification and gamma distributions: Comparison between 2D-Array measured data and the corresponding exported data coming from the treatment planning system. (2D-Array measurements were at zero angel degree) 
Kasr-El-Aini Journal Of Clinical Oncology And Nuclear Medicine

Tamer Dawod et al.

Table 1: The measured Total Scatter Factors (TSCFs), Collimator Factors (Sc) and calculated Phantom Scatter Correction Factors (PSCFs) for 6MV photon beam.

\begin{tabular}{|c|c|c|c|c|c|}
\hline Field size & Reading (water) cGy & TSCF & Reading (air) cGy & Sc & PSCF \\
\hline $1 \times 1$ & 41.3 & 0.596735 & 12.73 & 0.222 & 2.685 \\
\hline $2 \times 2$ & 54.04 & 0.780812 & 45.85 & 0.801 & 0.975 \\
\hline $3 \times 3$ & 57.05 & 0.824303 & 53.31 & 0.931 & 0.886 \\
\hline $4 \times 4$ & 59.4 & 0.858257 & 54.86 & 0.958 & 0.896 \\
\hline $5 \times 5$ & 61.51 & 0.888744 & 55.44 & 0.968 & 0.918 \\
\hline $6 \times 6$ & 63.39 & 0.915908 & 55.92 & 0.976 & 0.938 \\
\hline $8 \times 8$ & 66.66 & 0.963156 & 56.72 & 0.990 & 0.972 \\
\hline $10 \times 10$ & 69.21 & 1 & 57.27 & 1.000 & 1.000 \\
\hline $12 \times 12$ & 71.29 & 1.030053 & 57.73 & 1.008 & 1.022 \\
\hline $15 \times 15$ & 73.84 & 1.066898 & 58.19 & 1.016 & 1.050 \\
\hline $18 \times 18$ & 76.13 & 1.099986 & 58.65 & 1.024 & 1.074 \\
\hline $20 \times 20$ & 77.35 & 1.117613 & 59.2 & 1.034 & 1.081 \\
\hline $25 \times 25$ & 80.26 & 1.159659 & 61.09 & 1.067 & 1.087 \\
\hline $30 \times 30$ & 82.76 & 1.195781 & 62.24 & 1.087 & 1.100 \\
\hline $35 \times 35$ & 84.77 & 1.224823 & 63.23 & 1.104 & 1.109 \\
\hline $40 X 40$ & 85.96 & 1.242017 & 63.52 & 1.109 & 1.120 \\
\hline
\end{tabular}

\section{DISCUSSION}

\section{Commissioning data:}

Commissioning of a conventional treatment planning system requires both non-dosimetric and dosimetric tests $^{31,32}$. Non-dosimetric tests include such items as image registration and conversion, image and isodose display, and hardcopy devices. Dosimetric tests include comparisons between measurements and planning system predictions of, for example, depth doses, open and wedge beam profiles, and handling of inhomogeneities. Both measurements and dose calculations must be accurate, if the two are to match. Unlike conventional systems, IMRT planning systems must account for the additional complexity due to modulation of the fluence distribution. In general, the more complex the modulations the greater are the additional uncertainties that are introduced above those that already exist for conventional field calculations. So, by comparing the previously presented commissioning data which carried out on Artiste Linac with many similar published data coming from other Linacs (Elekta). We found that there are some slightly differences in most collected and compared data that specially related to MLC characteristics. The reason for this difference is coming from the different leaf width and high number of leaves (MLC) which is 160 leaves on Artiste Linac instead of 80 or 120 leaves for Elekta Linac. The narrow MLC openings required to deliver highly modulated fields can produce substantial dosimetric error ${ }^{33-35}$.

\section{IMRT verification:}

Our results demonstrate that the 2D-Array can be used to accurately and efficiently verify the dosimetry of IMRT treatment delivery. The setting of MapCheck comparison tolerance criteria is challenging and needs to take into account the uncertainties of the intensity-map measurements, the MLC calibration, radiation delivery and treatment plan dose calculation. Van Dyke et al..$^{31}$ and the AAPM task group $53^{36}$ suggest criteria of $3 \%$ of dose difference and 4 and $3 \mathrm{~mm}$, respectively, for dosimetry of conformal therapy beam.

Our tolerances between measurements and calculations are set at $3 \% / 3 \mathrm{~mm}$. The disagreement between the measured and calculated dose maps increases generally with the number of segments per beam and with the use of small segments delivering an appreciable fraction of the MUs.

For IMRT treatments, this becomes impractical and more elaborate QA measures are therefore necessary. When carrying out commissioning data and QA measurements for IMRT fields, it is preferable to use the MU that will be delivered for the patient treatment, since this will affect the MU per segment for step-and- 
shoot technique. Individual field checks may be either quantitative or qualitative and are generally performed using diode arrays. This is completely agreed with the published data that reported by McNair HA et al. ${ }^{37}$.

\section{CONCLUSION}

Modern IMRT methods are maturing and are being embraced by the medical community as a way to improve dose control and to improve patient quality of life. The pioneers in this field have put considerable effort into making the initial laborious process more efficient for clinical use. The year 2000 has seen the first major symposium on community-based IMRT implementation.

IMRT requirements differ somewhat from conventional treatment requirements. IMRT requires multiple segments for building its fluence maps. Accuracy in planning and delivery depends on multiple factors from hardware to calculation algorithms. The concept of integral dose gains importance with the use of MLCs and the delivery of a large number of MUs. MLC evaluations can include looking at conventional specifications as applied to IMRT, such as leakage without backup jaws over a region.

Dosimetry involves more than accounting for delivered dose. It involves ensuring that the MLC shapes are accurate and are assigned to the correct patient. Any changes in shaping must be incorporated into the field sequence correctly. The information system should be implemented in a way that ensures good quality assurance practices. The quality assurance procedures to check each IMRT patient with phantom measurement before the first setting of treatment are demanding and workload intensive.

\section{REFERENCES}

1. Inal A, Akkurt I. Dosimetric verification of calculation and measurements in radiotherapy using IMRT phantom. Sci. Res.Essays 2010 May 18;5(10):1213-5.

2. Yu CX, Amies CJ, Svatos M. Planning and delivery of intensity-modulated radiation therapy. Med.Phys. 2008;35(12):5233-41.

3. Stathakis S, Esquivel C, Gutierrez A, Buckey CR, Papanikolaou N. Treatment planning and delivery of IMRT using 6 and $18 \mathrm{MV}$ photon beams without flattening filter. App1/Radiat.Isotopes 2009;67(9):1629-37.

4. CarlsonD. Intensity modulation using multileaf collimators: Current status. Med.Dosimetry 2001;26(2):151-6.

5. Carlson DJ, Yang CCJ, Raben A. The First Community Hospital IMRT Symposium and Workshop-Monmouth Medical Center. Concord, CA: Siemens-OCSG; 2000.
6. Teh BS, Woo SY, Butler EB. Intensity Modulated Radiation Therapy (IMRT): A new promising technology in radiation oncology. Oncologist 1999;4(6):433-42.

7. Ezzell GA, Galvin JM, Low D, Palta JR, Rosen I, Sharpe $\mathrm{MB}$, et al. Guidance document on delivery, treatment planning and clinical implementation of IMRT: Report of the IMRT subcommittee of the AAPM radiation therapy committee. Med.Phys. 2003;30(8):2089-115.

8. Bortfeld T. Optimized planning using physical objectives and constraints. Semin.Radiat.Oncol. 1999;9(1):20-34.

9. LoSasso T, Chui CS, Ling CC. Physical and dosimetric aspects of a multileaf collimation system used in the dynamic mode for implementing intensity modulated radiotherapy. Med.Phys. 1998;25(10):1919-27.

10. Jayaraman S, Lanzl LH. Clinical radiotherapy physics: Basic physics and dosimetry. 1st ed. CRC Press; 1996.

11. Declich F, Fumasoni K, Mangili P, Cattaneo GM, Iori M. Dosimetric evaluation of a commercial 3-D treatment planning system using Report 55 by AAPM Task Group 23. Radiother.Oncol. 1999;52(1):69-77.

12. Protocol for the dosimetry of X-and gamma-ray beams with maximum energies between 0-6 and $50 \mathrm{MeV}$. Phys. Med.Biol. 1971;16(3):379-96.

13. Burlin TE. Radiation dosimetry. New York: Academic Press; 1968.

14. Central axis depth dose data for use in radiotherapy. A survey of depth doses and related data measured in water or equivalent media. Br.J.Radiol. 1983;Suppl. 17:1-147.

15. Aird EGA, Burns JE, Day MJ, Duane S, Jordan TJ, Kacperek A, et al. Central Axis Depth Dose Data for Use in Radiotherapy. Br.J.Radiol. 1996;Suppl. 25.

16. www.cmsrtp.com under Support/UserAccess/Xio Data Collection Tips for IMRT.

17. Bohsung J. The 2D-ARRAY seven29: A new way of dosimetric verification of IMRT beams. 2004.

18. Low DA, Harms WB, Mutic S, Purdy JA. A technique for the quantitative evaluation of dose distributions. Med. Phys. 1998;25(5):656-61.

19. Khan FM, Doppke KP, Hogstrom KR, Kutcher GJ, Nath $\mathrm{R}$, Prasad SC, et al. Clinical electron-beam dosimetry: Report of AAPM radiation therapy committee task group no. 25. Med.Phys. 1991;18(1):73-109.

20. Das IJ, Cheng CW, Watts RJ, Ahnesjö A, Gibbons J, Li XA, et al. Accelerator beam data commissioning equipment and procedures: Report of the TG-106 of the Therapy Physics Committee of the AAPM. Med.Phys. 2008;35(9):4186-215.

21. Radaideh KM, Alzoubi AS. Factors impacting the dose at maximum depth dose (dmax) for 6MV high-energy photon beams using different dosimetric detectors. Biohealth Sci. Bull. 2010;2(2):38-42.

22. Rafaravavy $\mathrm{R}$, Andriambololona $\mathrm{R}$ and Bridier $\mathrm{A}$. Study of dose distribution in high energy photon beam used in radiotherapy. Study of dose distribution in high energy photon beam used in radiotherapy. International Conference; Sep 10-15; Antananarivo, Madagascar; 2007. 


Vol. 8 | No. 1-2 $2012 \quad$ Tamer Dawod et al.

23. Mohan R, Jayesh K, Joshi R, Al Idrisi M, Narayanamurthy P, Majumdar SKD. Dosimetric evaluation of 120leaf multileaf collimator in a Varian linear accelerator with 6-MV and 18-MV photon beams. J.Med.Phys. 2008;33(3):114-8.

24. Cutanda Henriquez F, Vargas Castrillón ST. A study of beam homogeneity for a Siemens Primus linac. Australas. Phys.Eng.Sci.Med. 2007;30(2):147-51.

25. Khan FM. The Physics of radiation therapy. 3rd ed. Philadelphia, PA: Lippincott Williams \& Wilkins; 2003.

26. McKerracher C, Thwaites DI. Phantom scatter factors for small MV photon fields. Radiother. Oncol. 2008; 86(2):272-5.

27. Purdy JA. Intensity-modulated radiotherapy: Current status and issues of interest. Int.J.Radiat.Oncol.Biol.Phys. 2001;51(4):880-914.

28. Van Esch A, Bohsung J, Sorvari P, Tenhunen $\mathrm{M}$, Paiusco $\mathrm{M}$, Iori $\mathrm{M}$, et al. Acceptance tests and Quality Control (QC) procedures for the clinical implementation of intensity modulated radiotherapy (IMRT) using inverse planning and the sliding window technique: Experience from five radiotherapy departments. Radiother.Oncol. 2002; 65(1):53-70.

29. Depuydt T, Van Esch A, Huyskens DP. A quantitative evaluation of IMRT dose distributions: Refinement and clinical assessment of the gamma evaluation. Radiother. Oncol. 2002;62(3):309-19.
30. Boehmer D, Bohsung J, Eichwurzel I, Moys A, Budach V. Clinical and physical quality assurance for intensity modulated radiotherapy of prostate cancer. Radiother. Oncol. 2004;71(3):319-25.

31. Van Dyk J, Barnett RB, Cygler JE, Shragge PC. Commissioning and quality assurance of treatment planning computers. Int.J.Radiat.Oncol.Biol.Phys. 1993;26(2):261-73.

32. Kutcher GJ, Coia L, Gillin M, Hanson WF, Leibel S, Morton RJ, et al. Comprehensive QA for radiation oncology: Report of AAPM Radiation Therapy Committee Task Group 40. Med.Phys. 1994;21(4):581-618.

33. Liu C, Simon TA, Fox C, Li J, Palta JR. Multileaf collimator characteristics and reliability requirements for IMRT Elekta System. Int.J.Radiat.Oncol.Biol.Phys. 2008;71(1 Suppl. 1):S89-92.

34. Huq MS, Das IJ, Steinberg T, Galvin JM. A dosimetric comparison of various multileaf collimators. Phys.Med. Biol. 2002;47(12):N159-70.

35. Kwok CB, Lam G, El Sayed S. Suitability of using multileaf collimator (MLC) for photon field matching. Med.Dosimetry 2004;29(3):184-95.

36. Fraass B, Doppke K, Hunt M, Kutcher G, Starkschall $\mathrm{G}$, Stern R, et al. American association of physicists in medicine radiation therapy committee task group 53: Quality assurance for clinical radiotherapy treatment planning. Med.Phys. 1998;25(10):1773-829.

37. McNair HA, Adams EJ, Clark CH, Miles EA, Nutting CM. Implementation of IMRT in the radiotherapy department. Br.J.Radiol. 2003;76(912):850-6. 